\title{
Una opción para el paso a la minería subterránea masiva: la actual metodología aplicada en Chile del "block caving"
}

\author{
René Gómez ${ }^{(1)}$ y E. Labbé(2) \\ (1) Universidad de Concepción. Concepción, Chile. \\ regomez@udec.cl \\ (2) Codelco, División EITeniente. Rancagua, Chile. \\ elabbe@codelco.cl
}

\begin{abstract}
RESUMEN
El presente artículo es una revisión del método de explotación Block Caving enfocado en el desarrollo que ha tenido principalmente en los yacimientos de Chile, donde son identificadas las características particulares que engloban al método, así como estrategias de planificación llevadas a cabo para optimizar la explotación del recurso mineral en los nuevos entornos mineros. La explotación mediante Block Caving se realiza en yacimientos subterráneos masivos, generalmente cuando el costo debido a la profundidad del yacimiento o características particulares de éste no hacen viable la extracción mediante cielo abierto. La complejidad del método está asociada a la dinámica de hundimiento y colapso natural que se propaga en el macizo rocoso hacia la superficie consecuencia de la extracción del mineral. Actualmente han ido surgiendo yacimientos con nuevos desafíos como lo son menores leyes y mayores profundidades, siendo este último aspecto un parámetro crítico para el desarrollo del método como se ha venido realizando de manera convencional, enfrentándonos a condiciones de roca más competente, altos esfuerzos inducidos en los frentes de trabajo generando problemas geotécnicos, así como fragmentación más gruesa que genera dificultades en el sistema de manejo de materiales. Para abordar estas problemáticas, en el presente artículo se describen y analizan las diferentes estrategias que han sido evaluadas y aplicadas, así como su evolución en términos de estrategias de socavación inicial de la roca, variantes del método y pre-tratamiento del macizo rocoso para poder lograr la explotación.
\end{abstract}

Palabras claves: esfuerzos, fractura, hundimiento de bloques, minería subterránea.

\section{An option for the change to massive underground mining: the current Chilean methodology of block caving}

\begin{abstract}
The block caving method we review here is focused on Chilean deposits. Mining through block caving is applied to massive underground deposits when the open-pit method is not technically or economically viable. Block caving is a complex method ruled by the dynamics of caving and the breakage of the rock mass towards the surface due to ore extraction. Conventional block caving operations have been changing or depleting and consequently new deposits have appeared with new challenges, such as lower grades and greater depths. $A$ review of the block-caving mining method focused mainly on Chilean deposits is presented in this paper. The principal characteristics of the method as well as planning policies are reported and described. We analyze the strategies that have been applied and evaluated in recent years, as well as their evolution with the blasting method of the undercut level, modifications of the current method and pre-treatment of the rock mass to be able to achieve mining.
\end{abstract}

Keywords: stresses, fracture, block caving, underground mining. 


\section{Introducción a la minería de Block Caving}

Dentro de la minería subterránea masiva, los métodos de hundimiento (caving) son una opción preferible y viable cuando se buscan bajos costos y altos ritmos de producción, ambos factores importantes dada la mayor presencia de yacimientos de bajas leyes y roca mineralizada cada vez más competente (Flores 2014). Como principales desventajas de estos métodos se encuentran la alta inversión inicial y la baja flexibilidad a cambios en el diseño una vez que la operación ha comenzado (Castro 2006).

Existen diferentes métodos de hundimiento $y$ variantes tales como el Block Caving y Panel Caving cuando se explota desde un mismo nivel por periodo, también se encuentran alternativas como el Front Caving, Sublevel Caving y Inclined Block/Panel Caving, en explotaciones a través de subniveles a diferentes cotas de manera descendente (Figura 1) y en los últimos años se han incorporado las variantes de Minería Continua y Macro Bloques.

El Block Caving es un método masivo de gran escala en el cual el cuerpo mineralizado se hunde naturalmente tras iniciar el quiebre del macizo rocoso desde un nivel de hundimiento (Laubscher 1994, Brown 2007), esta técnica utiliza la gravedad en conjunto con esfuerzos internos de la roca para generar la fractura del macizo rocoso en fragmentos manejables que pasaran a través de los puntos de extracción para ser extraídos por un equipo de carga, comúnmente un Load Haul Dump (LHD) (Hamrin 2001). Por su parte, el Panel Caving funciona básicamente con el mismo mecanismo de colapso inducido al abrir un área en la base de la mineralización, pero en este caso se realiza un hundimiento continuo a través de paneles (Brown 2007), a diferencia del Block Caving donde el hundimiento es discreto a través bloques o áreas productivas. Los principales niveles requeridos para la explotación del método se ilustran en la Figura 2.
En la Figura 2, se observa de manera descendente el nivel de hundimiento requerido para iniciar el quiebre natural del macizo rocoso posterior a la aplicación de perforación y voladura, el diseño de este nivel puede presentar diferentes configuraciones, presentando una alta o baja socavación en función de la altura de la base de roca que se desee volar inicialmente. Este será un nivel temporal que después de su voladura desaparece producto de la extracción del mineral. Bajo este nivel y conectado a través de bateas de extracción se ubica el nivel de producción o extracción, donde los equipos de carga toman el mineral fragmentado desde los puntos de extracción ubicados bajo las bateas y lo descargan sobre piques de traspaso que conectan a un nivel de transporte. Para controlar la granulometría dentro del pique de traspaso se puede colocar en su entrada una parrilla la cual puede disponer de un martillo picador para romper los grandes bolos. También pueden existir equipos de machaqueo (trituración) primario en el interior de mina. En el nivel de transporte, dependiendo de la amplitud de la explotación minera, puede existir un nivel de transporte intermedio y posteriormente un nivel de transporte principal, esto con el fin de poder recolectar el mineral hacia un sector común para evacuar el mineral de la operación. Un ejemplo de lo anterior es posible observarlo en la operación de División El Teniente, Codelco, Chile. Si bien es un método masivo de alta productividad con operaciones que pueden superar las 120.000 toneladas por día (tpd), el factor clave en el manejo de materiales es la fragmentación natural que se genera dentro del macizo rocoso, parámetro a considerar también en el diseño minero.

Es posible distinguir en minería por hundimiento tres diferentes niveles de fragmentación: preexistente o in-situ, primaria y secundaria (Laubscher 1994, Brown 2007). La fragmentación in-situ corresponde a aquella presente naturalmente en el macizo rocoso como un conjunto de discontinuidades.
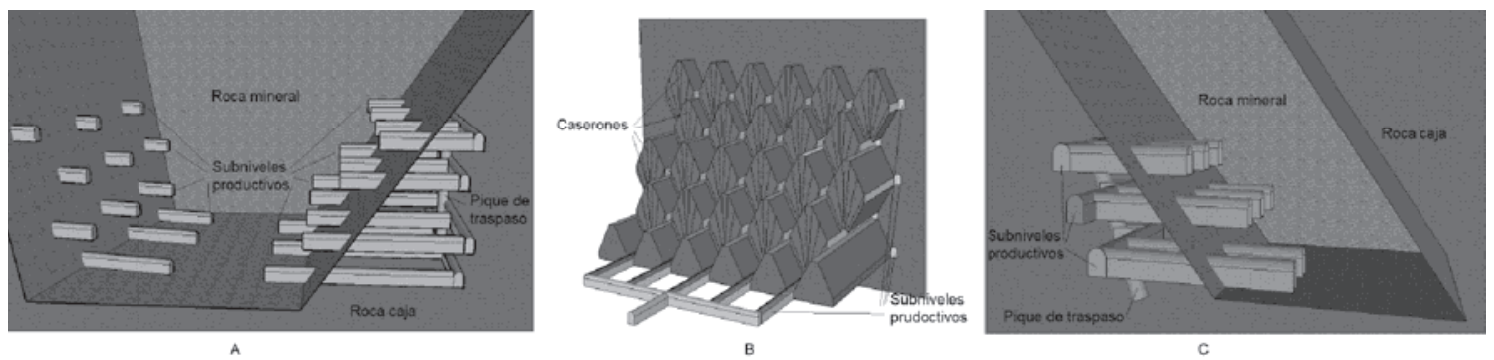

Figura 1. Variante de explotación a través de subniveles. A: Front Caving, B: Sublevel Caving, C: Inclined Block Caving. Figure 1. Sublevel alternatives in caving mining. A: Front Caving, B: Sublevel Caving, C: Inclined Block Caving. 


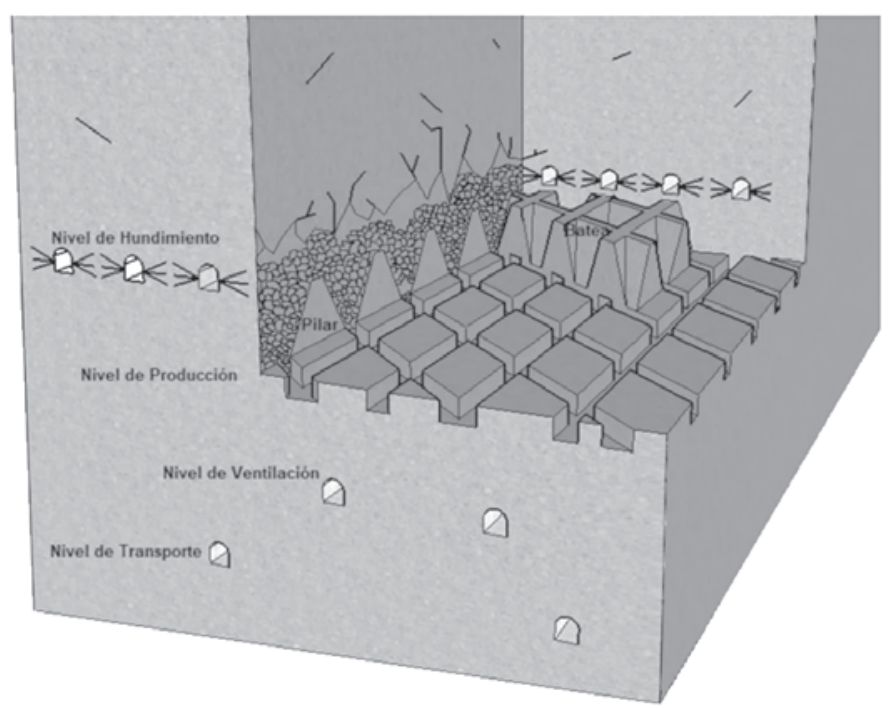

Figura 2. Esquema de explotación mediante Block/Panel Caving. Figure 2. Exploitation diagram by Block / Panel Caving.

Posteriormente, durante el proceso de hundimiento se observa la fragmentación primaria, originada por el desprendimiento de bloques desde el techo (cave back) por efecto de la relajación de los esfuerzos inducidos al generarse una gran área sin soporte. Finalmente, la fragmentación secundaria es generada en la columna de mineral hundido, asociada al movimiento de los bloques a través de la columna hasta llegar a los puntos de extracción (Brown 2007). Debido a la importancia de la fragmentación en el éxito de la operación se han realizado diversos estudios al respecto para poder predecirla (Eadie 2003, Pierce 2009, Pierce et al. 2010, Jakubec 2014, Brunton et al. 2016, Gómez et al. 2017).

Dificultades asociadas a la fragmentación son las colgaduras en piques, bateas y puntos de extracción ya sea por grandes bolos, alta sobrecarga y/o la presencia de material cohesivo como finos y humedad (Beus et al. 2001, Hadjigeorgiou y Lessard 2007, Kvapil 2008, Castro et al. 2016), así como también la presencia de grandes tamaños en los puntos de extracción que no pueden ser tomados por el equipo de carguío. La presencia de material fino genera dificultades relacionadas con la dilución, que si bien no es una complicación exclusiva de los métodos de hundimiento, posee diversas implicaciones a lo largo de las diferentes etapas de un proyecto minero, como en las etapas de elección de método, su selectividad, diseño minero, productividad, así como también en etapas posteriores durante el procesamiento de minerales y sustentabilidad (Laubscher 2000). Esta dilución dentro de una explotación de caving puede provenir de las paredes del cuerpo mineralizado y/o de la parte superior, ya sea desde sectores previamente explotados o desde roca estéril fragmentada. Debido a sus implicancias también es una variable que ha sido altamente estudiada por diversos autores (Lorig 2004, Susaeta et al. 2008, Hashim 2011, Castro y Paredes 2014, Castro y Pineda 2015).

El incremento de la profundización de las operaciones debido al continuo agotamiento de las reservas superficiales es una realidad de la industria minera, con algunos ejemplos de mega proyectos en Chile como Mina Chuquicamata Subterránea y Nuevo Nivel Mina, evidenciando las mayores profundidades a las cuales la minería de hundimiento se está viendo enfrentada. En la Figura 3 se resumen las tendencias en cuanto a la profundización de diferentes proyectos de hundimiento masivo alrededor del mundo.

La profundización en los yacimientos va a incrementar la altura de columna de material quebrado generada en el proceso de hundimiento (desde nivel de producción hasta la superficie) aumentando los esfuerzos asociados a la sobrecarga del material. Existen antecedentes del colapso de áreas productivas como es el caso de la mina DOZ, explotada en Indonesia, donde el peso de la columna generó la pérdida de zonas las cuales contenían entre el $15 \%$ y $30 \%$ de las reservas teóricas. En aquel caso, fue necesario aplicar una técnica de recuperación de pilares con la que se logró obtener entre un $85 \%$ y $90 \%$ de la zona colapsada (Sahupala et al. 2008).

Dentro de los principales riesgos asociados a la minería de hundimiento están los estallidos de roca, bombeos de barro, estallidos de aire y colapso de infraestructuras mineras, generando situaciones de potencial riesgo para el personal y equipos, como también la pérdida de la continuidad del proceso productivo de la operación minera.

Los estallidos de roca son uno de los grandes problemas presentes en minería subterránea principalmente en roca competente bajo altas condiciones de esfuerzos, fenómeno correspondiente a una rotura violenta y espontánea de roca cercana a los límites de una excavación producto de una alta concentración de esfuerzos, situación que puede ser inducida por un evento sísmico. Los estallidos de rocas se consideran como condiciones inestables de energía dentro del macizo rocoso (Laubscher 2000).

Por otro lado, el bombeo o estallido de barro se conoce como una entrada repentina y violenta de una mezcla compuesta de agua y material fino hacia labores mineras subterráneas (Butcher et al., 2000, Laubscher 2000), existen registros de diversos accidentes a diferentes magnitudes ocurridos en minería de hundimiento (Syaifullah 2006, Samosir et el 2008, 


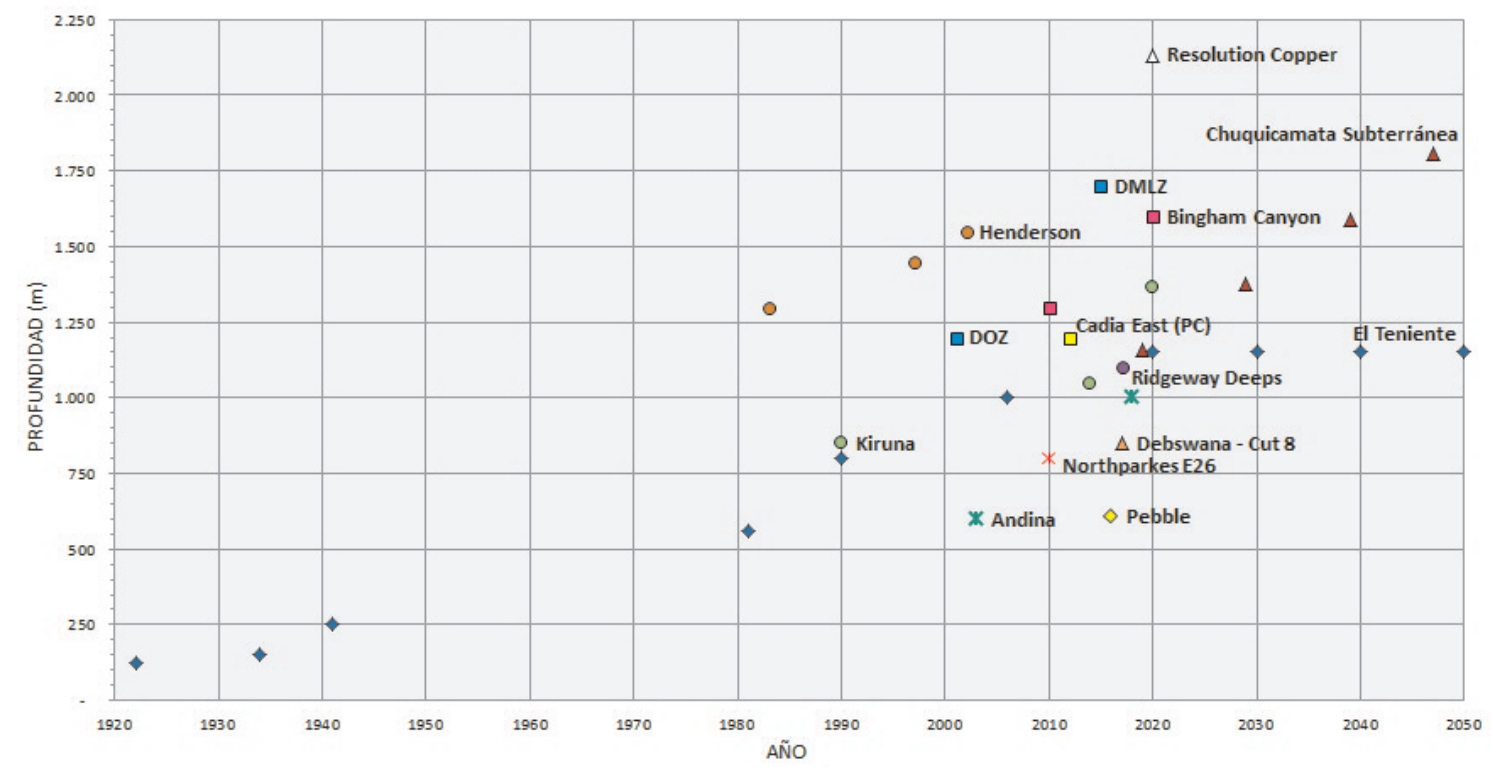

Figura 3. Profundización de proyectos en minería masiva de caving (Labbé 2014).

Figure 3. Deepening in massive caving mining (Labbé 2014).

Codelco 2010, Briceño et al. 2016, Vallejos et al. 2017). Butcher et al. (2000) menciona cuatro factores que al estar presentes pueden generar este tipo de evento: material formador de barro, agua, una perturbación y un punto de descarga del bombeo.

Mientras que un estallido de aire es el resultado de un efecto pistón generado por el colapso violento de un gran volumen de roca dentro de una cavidad subterránea. La escala del estallido dependerá del tamaño de la cavidad, el volumen de roca y la velocidad del colapso. Este fenómeno ha sido frecuente en operaciones de Block Caving a profundidades variables, aunque la mayoría de los eventos han ocurrido cercanos a la superficie (Laubscher 2000, Brown 2007). Un registro de este tipo de evento ha sido presentado por De Nicola y Fishwick (2000), donde un violento estallido de aire fue registrado en la mina Salvador, Chile.

El colapso geomecánico, por su parte, consiste en un descenso paulatino de un área, generalmente en el nivel de producción de una operación de Block Caving, con daño observado en los pilares corona (Crown Pillar) y calle/zanja, cuya expresión máxima es el cierre total de las galerías afectadas, reduciendo el área productiva debido a la pérdida de los accesos a ella. Tanto los riesgos particulares asociados a los métodos de hundimiento como la propia dinámica de la explotación se generan debido a la metodología de propagación del hundimiento en el macizo rocoso.

Las dimensiones asociadas al método requeridas para poder generar la desestabilización natural del macizo rocoso conlleva a que los yacimientos aplicados sean frecuentemente pipas geológicas, pórfidos y mineralizaciones masivas, teniendo presente que puedan sustentar un gran área. Una situación preferente se genera cuando se tiene una roca poco competente la cual será favorable para la propagación del hundimiento, situación que se puede ver favorecida dependiendo del patrón estructural presente.

Debido a los altos ritmos de explotación, tecnología disponible y la fragmentación natural de gran parte de la roca mineral dentro de la excavación, se considera como el método de explotación subterráneo con el menor costo operacional cercano a los 510 USD/ton. Ahora bien, es requerida una alta inversión inicial y tiempos de desarrollo extensos previo al inicio de la extracción de mineral, del cual se estiman recuperaciones mineras del orden del $70 \%$ al $90 \%$ de las reservas disponibles.

\section{Minería de Block Caving en Chile}

Chile se caracteriza por ser un país que desarrolla activamente la industria minera, generando un importante ingreso al PIB nacional, asociado principalmente a la minería metálica y en particular a la explotación de cobre. Además, cuenta con el 29,2\% de las reservas mundiales de cobre (Ministerio de Minería 2016) cuya explotación genera como producto concentrados $(30 \% \mathrm{Cu})$ y cátodos $(99,99 \% \mathrm{Cu})$ de éste metal. 
Dentro de los principales yacimientos explotados y explorados en Chile destacan los pórfidos, con un $35 \%$ de la exploración, los IOCG (óxidos de hierro con cobre y oro) con un $23 \%$, un $20 \%$ de yacimientos indeterminados y un $10 \%$ en yacimientos hidrotermales HS, concentrando en la zona norte del país el $86 \%$ de la exploración minera (Cochilco 2014). El cobre presente en estos yacimientos se encuentra principalmente en menas de Calcopirita, Cuprita, Covellina, Calcosina, Bornita, Azurita, Malaquita, Crisocola y Calcantita.

Actualmente en el país existen tres operaciones con este método o su variante Panel Caving, cuyos yacimientos cuentan con leyes cercanas al 0,7\% $0,9 \% \mathrm{Cu}$. Estas operaciones pertenecen a la empresa estatal Codelco, siendo una de las principales empresas que explotan cobre en el mundo tanto en operaciones superficiales como subterráneas. Dentro de las operaciones de Codelco que utilizan actualmente el método de hundimiento, se encuentran División Salvador, División Andina y División El Teniente (Coldelco 2018). Actualmente se encuentra en proceso de transición cielo abierto/subterránea el proyecto Mina Chuquicamata Subterránea a través del método de Block Caving con la variante de Macro-bloques.

Entre paréntesis en la Figura 4 se pueden observar los años de inicio de cada explotación y fundición (para el caso de Ventanas sólo es fundición).
Dentro de los futuros proyectos subterráneos en minería de Block Caving en Chile está la transición de División Chuquicamata que posee fecha de inicio planificada para el año 2019, esperando extender la vida útil del yacimiento en 40 años a través de tres niveles productivos a diferentes cotas consecutivamente $y$ alcanzando una producción estimada de 140.000 tpd en régimen. Además, se está desarrollando la profundización de la operación de División El Teniente, con fecha de inicio estimada para el 2023, esperando extender la vida útil del yacimiento en 50 años y alcanzar un ritmo de explotación de 137.000 tpd una vez que entre en régimen.

Ambos proyectos son considerados de reposición, con el objetivo de recuperar las capacidades productivas de las minas que se han visto disminuidas por efecto del deterioro de la ley de los yacimientos. Si agregamos además los proyectos de expansión de distintas empresas mineras y el inicio de otras nuevas operaciones, el escenario país revela para el año 2025 una proyección de aumento importante en el consumo eléctrico esperado entre las operaciones vigentes y los proyectos futuros según su tipo.

Actualmente, el consumo energético asociado a la minería en Chile es cercano a los 22 TWh anual, estimando aumentar a los 39,5 TWh para el año 2025. La distribución de consumo de las diferentes actividades dentro de la industria minera se divide en un $51 \%$ en

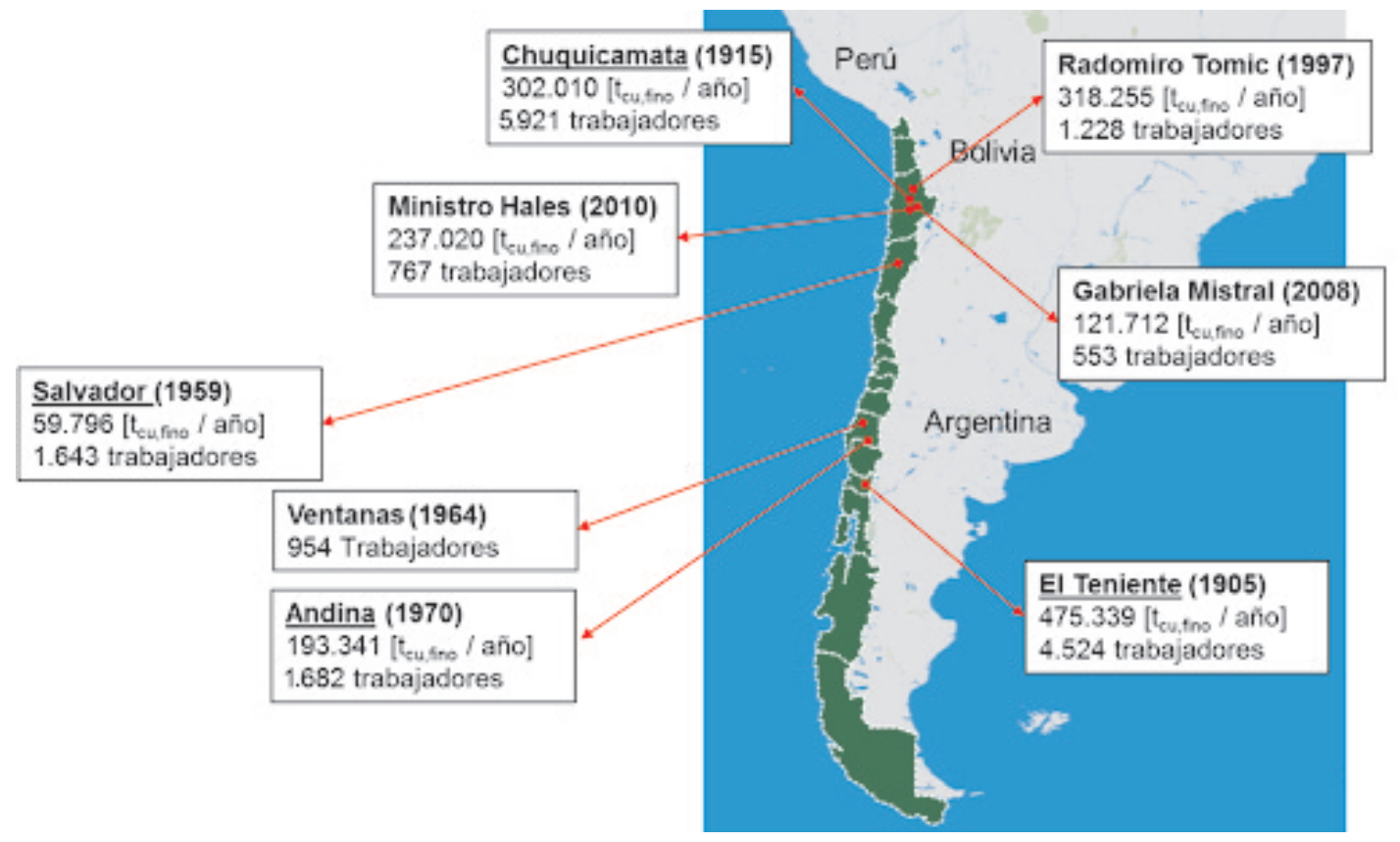

Figura 4. Operaciones de Codelco, Chile.

Figure 4. Operations of Codelco, Chile. 
la concentradora donde ocurren los principales procesos de reducción de tamaño, $24 \%$ en los procesos de lixiviación, SX y EW, 7\% en fundición, $6 \%$ en desalinización e impulsión de agua de mar y el porcentaje restante se reparte entre la corta, mina subterránea, refinería y servicios (Cochilco 2014). Una de las principales razones por las cuales no siempre se llega al producto final (cátodo) es debido al costo de la energía en Chile, cercano a los 0,15 USD/kWh, lo que eleva los costos operacionales frente a fundiciones ubicadas en otras regiones del mundo como China con un costo energético cercano al 0,08 USD/kWh (Emol 2017), entre otros factores.

\section{Estrategia y planificación en la explotación}

Cuando se aplica el método de Block Caving, el área basal del cuerpo mineralizado se subdivide en bloques y el área socavada se incrementa de manera discreta, en función de las necesidades de incorporar nuevos bloques en producción. En cambio, si se utiliza Panel Caving, la socavación del cuerpo mineral sigue un patrón relativamente continuo, típicamente dado por una tasa de área socavada por período, lo que hace que la estrategia de incorporación de área productiva sea mucho más flexible en este método que en Block Caving (Flores 2005). Como resultado de lo anterior, en Panel Caving se tendrá un frente de hundimiento que se moverá atravesando el cuerpo mineralizado guiado por la dirección del avance de la socavación (Brown 2007).

\section{Mecanismos de hundimiento del macizo rocoso}

Al iniciar la explotación y realizar el corte basal en el nivel de hundimiento comienza el colapso natural de macizo rocoso hasta propagarse el quiebre de la roca a la superficie. En la dinámica del proceso ocurre: Desconfinamiento o Caving gravitacional, inducido por discontinuidades; Stress Caving, el cual envuelve falla de corte en discontinuidades y fracturamiento de roca; Hundimiento y subsidencia (Duplantic and Brady 1999).

Al realizar una excavación subterránea hay una serie de factores que influirán en el grado de estabilidad de la excavación, entre ellos se pueden distinguir factores geométricos, estructurales y propiedades de la roca. Es así como en minería se identifican gráficos de estabilidad de excavaciones que proponen diversos autores en los cuales se consideran estas características usualmente utilizando algún factor de calidad del macizo rocoso (Mathews et al. 1981, Carter
1992, Lang 1994). Existen gráficos de estabilidad aplicados específicamente a operaciones de Block Caving como el presentado por Laubscher (1994). Con ello es posible contar con una primera aproximación del área (en planta) requerida para que el hundimiento se propague de forma natural.

A medida que va progresando el desplome del techo de la cavidad abierta, van alterandose los esfuerzos inducidos sobre este, lo que impacta en el grado de fragmentación primaria que se va generando; de igual modo, al ir aumentando la altura de extracción de mineral, los fragmentos de roca que caen en la columna de material quebrado estarán un mayor tiempo de residencia dentro de éste, aumentando la probabilidad de sufrir un evento de quiebre y se verán sometidos a una mayor sobrecarga al ir fluyendo a través de la columna, aumentando el grado de fragmentación secundaría. Al ir progresando la extracción de mineral, el tamaño de los bloques que aparecen en los puntos de extracción tiende a disminuir en el tiempo, favoreciendo el manejo de materiales posterior y disminuyendo las interferencias asociadas a grandes fragmentos. Un esquema de una cavidad generada por Block Caving se puede identificar en la Figura 5. El mineral es extraído a través de bateas ubicadas en la base mientras se va generando el hundimiento del techo de la cavidad a medida que se va fragmentando el macizo rocoso.

Dependiendo de la geometría de la cavidad, los esfuerzos inducidos en el techo pueden ser de tracción o compresión. Si los esfuerzos son bajos o de tracción, el quiebre y caída de fragmentos de roca ocurre debido a la presencia de un arreglo estructural que conforme bloques que puedan caer verticalmente (Brown 2007), esto se conoce como mecanismo de hundimiento por gravedad o relajación de esfuerzos. En caso contrario para altos esfuerzos, el hundimiento de los bloques alrededor de la excavación se debe a la ruptura frágil de la roca o deslizamiento de discontinuidades, mecanismo conocido como esfuerzo de hundimiento (Duplantic and Brady 1999).

\section{Estrategias de socavación}

Por estrategia de socavación se hace referencia a la secuencia de construcción de las labores productivas, es decir, la temporalidad de la tronadura de socavación relativa al desarrollo de las labores en los niveles de hundimiento y producción, y a la apertura de las bateas. Diversos autores (Laubscher 2000, Wattimena 2003, Brown 2007) coinciden en que las estrategias de socavación más importantes son:

- El hundimiento posterior o convencional 


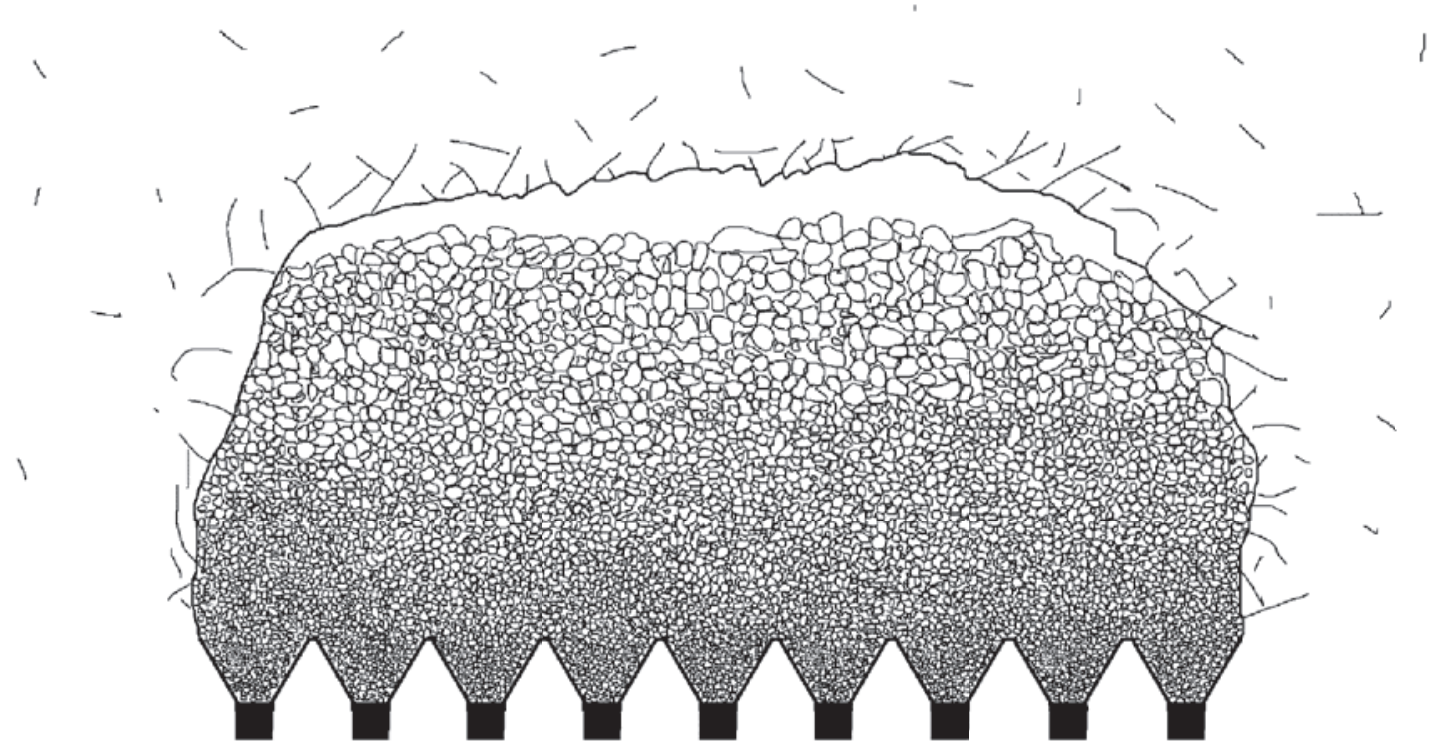

Figura 5. Esquema de cavidad generada en un Block Caving en propagación.

Figure 5. Cavity diagram generated in a Block Caving under propagation.

- El hundimiento avanzado

- El hundimiento previo

\section{Hundimiento convencional}

La secuencia constructiva de labores utilizada en esta metodología se muestra en la Figura 6 y se detalla a continuación (Karzulovic 1998, Rojas et al. 2000):

- Desarrollo labores en Niveles de producción y de Hundimiento.

- Apertura de bateas de extracción.
- Tronadura de socavación en el nivel de hundimiento, avanzando con el frente de hundimiento hacia las bateas abiertas.

- Inicio de la extracción.

En este escenario, prácticamente no hay diferencia entre el frente de extracción, limitado por la última línea de bateas incorporadas a producción y el frente de socavación asociado al avance del corte basal en el nivel de hundimiento. Dada la secuencia constructiva, al desarrollarse las labores del nivel de producción por delante de la frente de hundimiento, éstas se ven sometidas a la zona de altos esfuerzos inducidos,

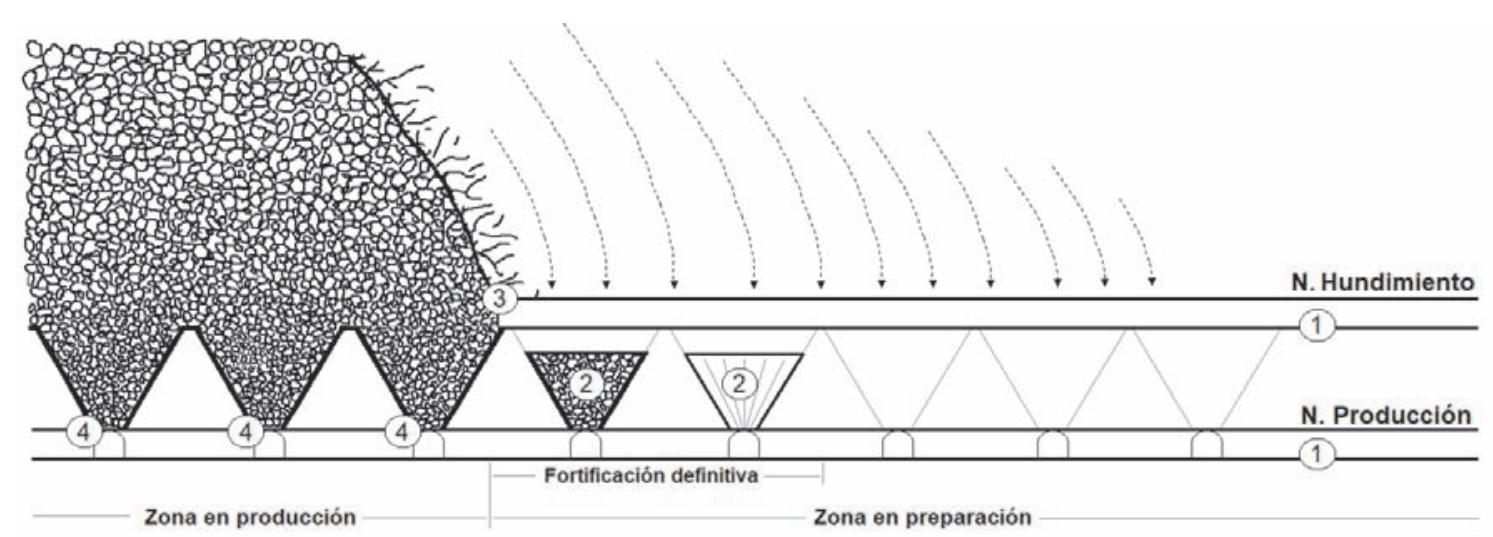

Figura 6. Secuencia constructiva en el método de hundimiento convencional. Figure 6. Constructive sequence in the conventional sinking method. 
indicadas por flechas discontinuas en la Figura 6. Esto generará un primer impacto en el pilar corona del nivel de producción, el cual se verá intensificado una vez que se produzca la apertura de bateas.

Con respecto a la utilización de esta estrategia de socavación, Butcher (2000) sugiere que, como guía general, su uso debería ser analizado seriamente si por ejemplo la profundidad de la faena es mayor a 500 metros o bien si el área de socavación tiene un radio hidráulico mayor a 17 metros, debido al daño generado por los altos esfuerzos inducidos (abutment stress) en el nivel de producción.

\section{Hundimiento previo}

La aparición de técnicas de socavación alternativas a la metodología convencional es el resultado de una serie de investigaciones y pruebas cuyos orígenes se remontan al año 1970 (Karzulovic 1998). En aquellos años, ya se consideraba el potencial uso de excavaciones de protección, que mediante la generación de sombras de esfuerzos, podrían disminuir el riesgo de ocurrencia de inestabilidades geotécnicas asociadas a concentraciones de esfuerzos. Posteriormente, a comienzos de los años 80, y luego de diversos estudios de aplicabilidad de este concepto en la mina El Teniente, se definió el Ilamado "hundimiento previo", en donde la socavación del bloque estaría adelantada respecto a la preparación de las labores en el nivel de producción.

A diferencia de la estrategia convencional, y como se muestra en la Figura 7, en esta estrategia la secuencia constructiva de labores es la siguiente (Karzulovic 1998, Rojas et al. 2000):
- Preparación de labores en el nivel de hundimiento.

- Voladura de socavación en el nivel de hundimiento avanzando con el frente de socavación hasta alcanzar una cierta distancia por delante del futuro frente de extracción.

- Preparación de labores en el nivel de producción.

- Apertura de bateas bajo área socavada.

- Inicio de la extracción.

La principal característica del método es el desarrollo de todas las labores del nivel de producción bajo área completamente socavada, hasta una distancia de seguridad por detrás del frente de socavación. De este modo, la concentración de esfuerzos que se forma por delante y hacia abajo de dicho frente tiene un efecto mucho menor sobre las instalaciones del nivel de producción, tal como se aprecia por las líneas discontinuas en la Figura 7.

Brown (2007) menciona que la distancia de seguridad puede estimarse utilizando la regla de los $45^{\circ}$, asumiéndola idéntica a la separación entre los niveles de producción y de hundimiento. Sin embargo, el mismo autor plantea que en algunas minas como Mina Esmeralda de División El Teniente, ha sido más satisfactorio mantener una mayor distancia que la recomendada por esta regla, llegando a utilizar una franja de seguridad de 22,5 metros, teniendo una separación entre niveles de no más de 18 metros.

\section{Hundimiento avanzado}

Diversos autores mencionan que el hundimiento previo puede ser considerado como una variante del hundimiento avanzado (Trueman et al. 2002,

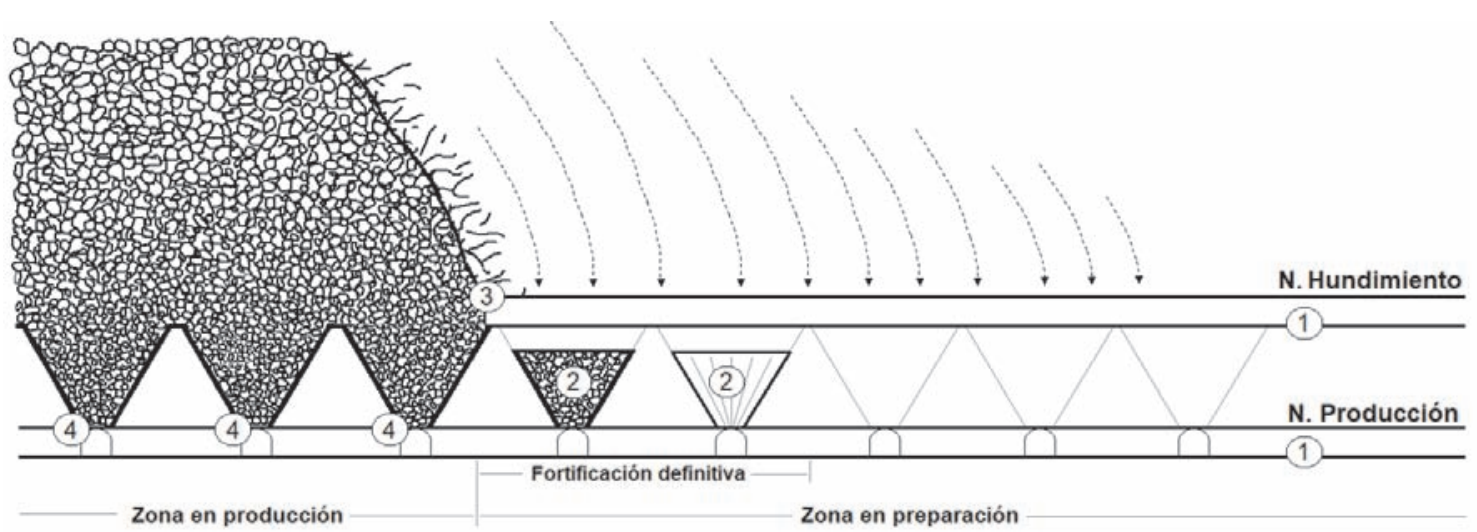

Figura 7. Secuencia constructiva del método hundimiento previo. Figure 7. Constructive sequence of previous subsidence method. 
Wattimena 2003, Brown 2007). En este método, la socavación en el nivel de hundimiento se realiza sobre un nivel de producción parcialmente desarroIlado. La secuencia constructiva de labores se detalla a continuación (Karzulovic 1998):

- Desarrollo de las labores del nivel de hundimiento, y de algunas del nivel de producción. En general solamente se construyen las calles de producción.

- Voladura de socavación en el nivel de hundimiento avanzando con el frente de socavación hasta alcanzar una cierta distancia por delante del futuro frente de extracción.

- Se desarrollan las restantes labores del nivel de producción en el sector bajo el área socavada.

- Se realiza la apertura de las bateas.

- Se inicia la extracción.

Las bateas son preparadas en una zona relajada desde el punto de vista geotécnico (ver Figura 8), usualmente considerando como mínima distancia de seguridad, medida en la horizontal, entre el frente de socavación y la línea de incorporación de bateas, una distancia dada por la regla de los $45^{\circ}$ (Brown 2007).

Actualmente, la tendencia en el diseño de Block y Panel Caving es seleccionar la estrategia de socavación avanzada, para aprovechar sus ventajas principalmente en cuanto a la reducción de los niveles de fortificación y la mayor productividad del método (Brown 2007). Basta con revisar en la literatura algunos diseños propuestos en los últimos años para confirmar lo anteriormente expresado: Premier Diamond Mine (Bartlett y Croll 2000), Palabora (Calder et al. 2000), Northparkes E26 lift 2 (Silveira 2004) y Argyle (Undercutting Workshop 2008) y Freeport DOZ (Undercutting Workshop 2008).
Pese a lo anterior, Bartlett y Croll (2000) plantean que la continua profundización de la minería de hundimiento incrementará de tal manera las magnitudes de los esfuerzos, que para evitar niveles de daño considerables en la infraestructura del nivel de producción, la mejor opción para controlar el daño consistirá en implementar un hundimiento previo, en particular a profundidades cercanas a los mil metros. Lo anterior se reafirma en el trabajo de Beck et al. (2006), quien concluyó que en un ambiente de altos esfuerzos, emplear socavación avanzada se traducirá en elevados niveles de daño en el nivel de producción, por lo que la estrategia de socavación previa puede tener algunas ventajas, en particular en torno a los mil metros de profundidad.

\section{Geometría de socavación}

El diagrama de perforación utilizado para realizar el corte basal, define la geometría de la socavación vista en sección vertical. En la literatura, se reconocen tres técnicas principales: socavación mediante abanicos, corte horizontal angosto y corte inclinado angosto. Donde la variante geométrica relevante es la diferencia entre las alturas de socavación para cada caso.

A modo de ejemplo, en la mina ElTeniente, el diseño de explotaciones por Block Caving con extracción manual, hasta comienzos del año 1980 se utilizaban reglas empíricas que relacionaban la altura de socavación con la altura del bloque a hundir, o bien con la altura de la galería de hundimiento (Karzulovic 1998). Un caso interesante ocurrió en minas de asbesto en Zimbawe, en donde se obtuvieron idénticos resultados en fragmentación y hundibilidad al reemplazar

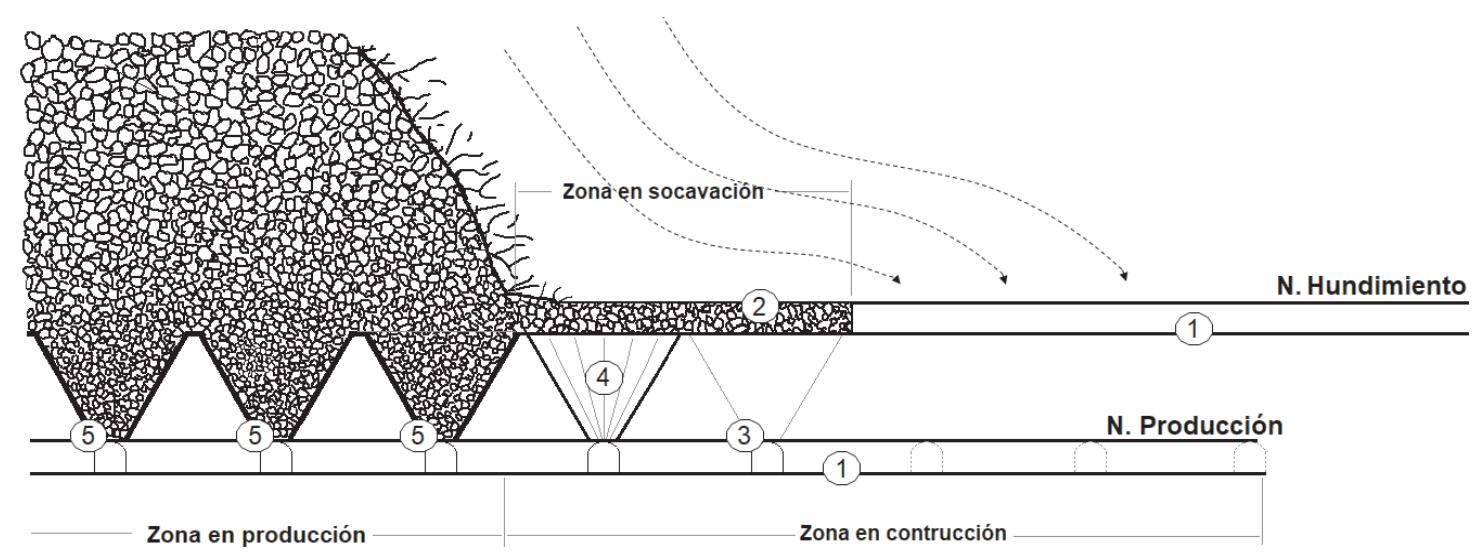

Figura 8. Secuencia constructiva de hundimiento avanzado.

Figure 8. Construction sequence of advanced subsidence. 
una altura de 20 metros, por una socavación de 3 metros de altura (Laubscher 2000), esto debido a que la altura de socavación no debiese influir en la desestabilización de la base para iniciar el desplome del techo de la cavidad generada. En la actualidad, se acepta que el principal motivo para utilizar mayores alturas de socavación es netamente para alcanzar mayores productividades iniciales (Butcher 2000), debido al mayor tonelaje fragmentado ya disponible para ser extraído.

La altura de socavación ha evolucionado en el tiempo, desde alturas medias en torno a los diez metros a bajas alturas, del orden de cuatro metros, asociadas a la implementación de hundimiento avanzado (Flores y Karzulovic 2002).

\section{Socavación en abanico}

Esta ha sido la manera más común de diseñar la perforación de socavación en la historia de las minas de Block Caving, siendo aplicada con todos los tipos de extracción: parrillas, scrapers y LHD (Butcher 2000). Se trata principalmente de una socavación alta, superando típicamente la altura de la galería. Entre las ventajas de esta técnica, se encuentran las siguientes:

- Se basa en un diseño relativamente flexible de la perforación a realizar, principalmente en cuanto a los equipos de perforación a utilizar y alturas a perforar.
- Dado que es posible aumentar la altura a socavar, es posible aumentar el tonelaje inicial asociado a la socavación.

- Es una metodología validada industrialmente, apoyada en diseños de socavación sobre los que se tiene la experiencia suficiente.

Mientras que, en cuanto a las desventajas del método, se destaca principalmente la formación de pilares post tronadura. Un ejemplo de este tipo de técnica se muestra en Figura 9.

\section{Socavación horizontal angosta}

En este caso se practica un corte bajo, realizado de manera horizontal desde las galerías de hundimiento, generando una altura de socavación similar a la altura de la galería. Esta técnica ha sido utilizada en la mina Esmeralda de EITeniente, donde las principales variantes que se han aplicado son las siguientes (Brown 2007):

- Perforación de la mitad del pilar de hundimiento desde cada una de las galerías adyacentes, método conocido como "John Wayne". Aquí se hace voladura desde ambas cajas de la galería de hundimiento.

- Perforación del pilar completo desde solo una galería de hundimiento (ver Figura 10). Voladura desde una caja de la galería de hundimiento.

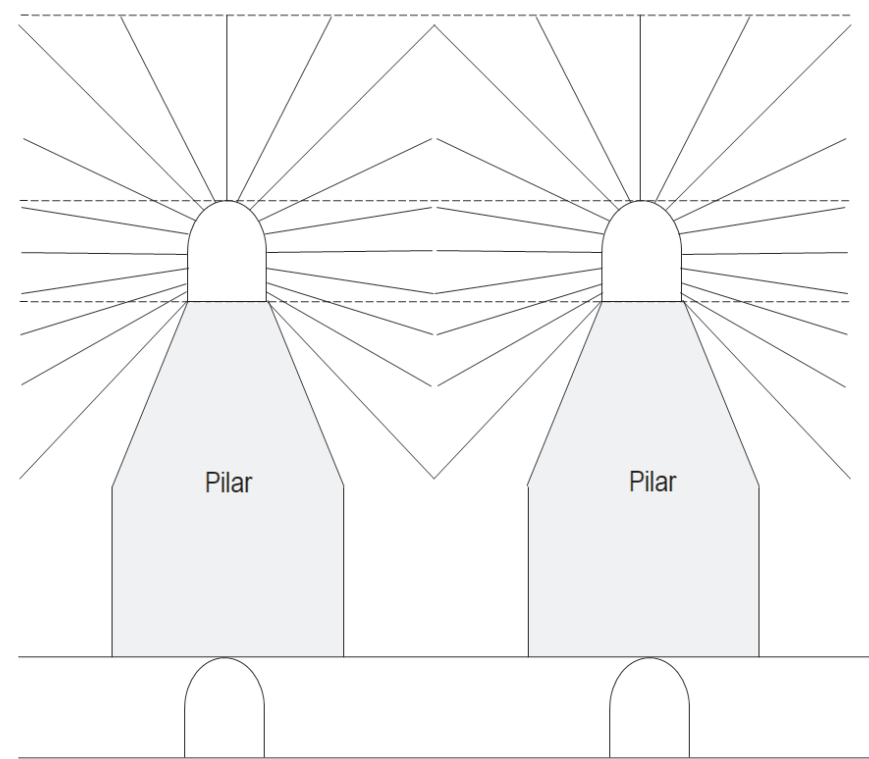

Límite superior

de socavación

Nivel

Hundimiento

Nivel

Producción

Figura 9. Socavación mediante abanicos.

Figure 9. Scouring with fans. 


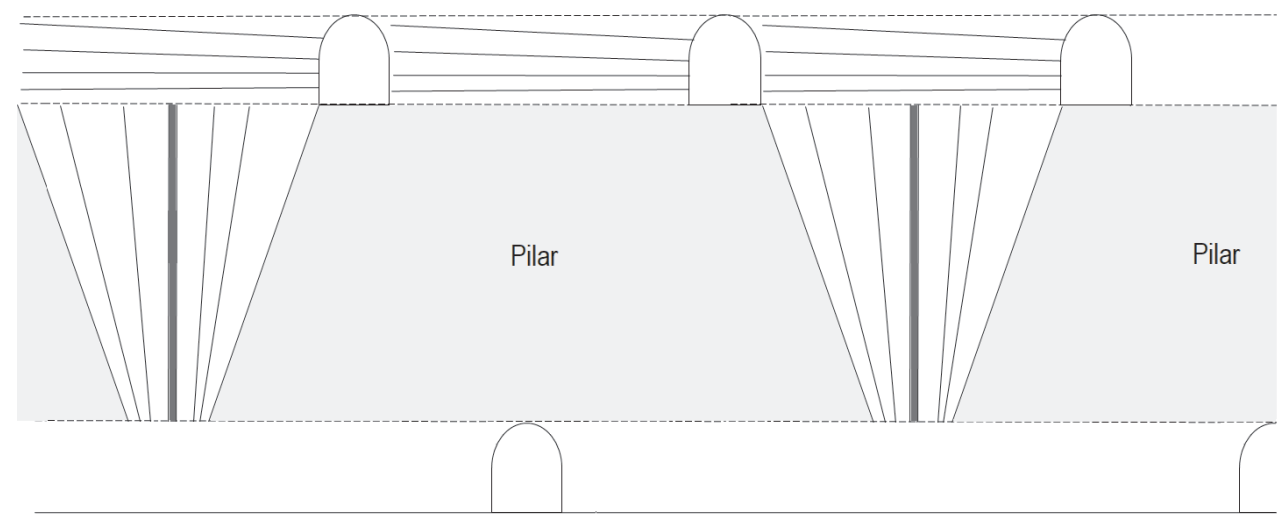

Nivel Hundimiento

Nivel Producción

Figura 10. Socavación horizontal angosta con perforación a pilar completo.

Figure 10. Narrow horizontal undercut with full pillar drilling.

- Perforación de tiros paralelos hacia el frente de socavación, desde una galería de conexión entre las calles de hundimiento.

Según Butcher (2000), este tipo de metodología de perforación es a menudo utilizada en niveles profundos principalmente debido a que genera mayores tasas de avance, dado que se requiere una menor perforación, y porque una menor altura de socavación produce menores esfuerzos inducidos.

Entre los principales aspectos negativos del método se encuentran (Karzulovic 1998, Laubscher 2000, Butcher 2000):

- La perforación horizontal tiene una probabilidad mayor que la inclinada de cerrarse producto de esfuerzos inducidos, debido a la verticalidad del esfuerzo principal mayor presente en el pilar a volar.

- Si el espaciamiento de puntos de extracción es grande (mayor a $15 \mathrm{~m}$ ), es mayor la probabilidad de inhibir el hundimiento.

- Una pobre geometría de la batea recolectora no permitirá el flujo de mineral.

- Este método, al estar asociado a un menor tonelaje inicial, no permitirá alcanzar altas tasas iniciales de producción.

Existe evidencia de que una socavación estrecha aumenta la probabilidad de ocurrencia de colgaduras en las bateas, fragmentación más gruesa, formación de planchones y de puntos de apoyo, lo que finalmente se traduce en interrupciones en el flujo de mineral.

\section{Socavación angosta inclinada}

El objetivo de esta metodología es generar la forma del pilar mayor a través de perforación inclinada, descendente $o$ ascendente, desde el nivel de hundimiento. La inclinación debe exceder el ángulo de fricción generado entre el material volado y la inclinación de dicho pilar, a fin de asegurar el flujo de mineral. En la actualidad, esta técnica de corte se está empleando en sectores profundos y con altos niveles de esfuerzos, como Palabora y Northparkes E26 (Brown 2007). Típicamente, las galerías de hundimiento se ubican en el mismo nivel, siendo dos las configuraciones constructivas más importantes (Laubscher 2000, Brown 2007):

- Construcción de dos galerías de hundimiento sobre cada batea, realizando perforación horizontal sobre ésta, y perforación inclinada ascendente sobre el mayor ápex formando una "V" invertida, tal como se muestra en la Figura 11.

- Construcción solo de una galería de hundimiento sobre cada batea. En este caso, solo se realiza perforación del tipo inclinada sobre el mayor ápex formando una "V" invertida.

Uno de los problemas que se puede llegar a presentar con esta técnica, son la generación de pilares remanentes en la parte superior del pilar mayor, lo que puede ser resuelto sobre perforando los tiros desde el nivel de hundimiento, o bien, agregando una galería en esa ubicación, lo que eleva los costos de preparación.

\section{Lineamientos del caving y estrategia de control}

Debemos recordar que el método por paneles induce la propagación del hundimiento a través de una socavación basal de la misma manera que lo hace por blo- 


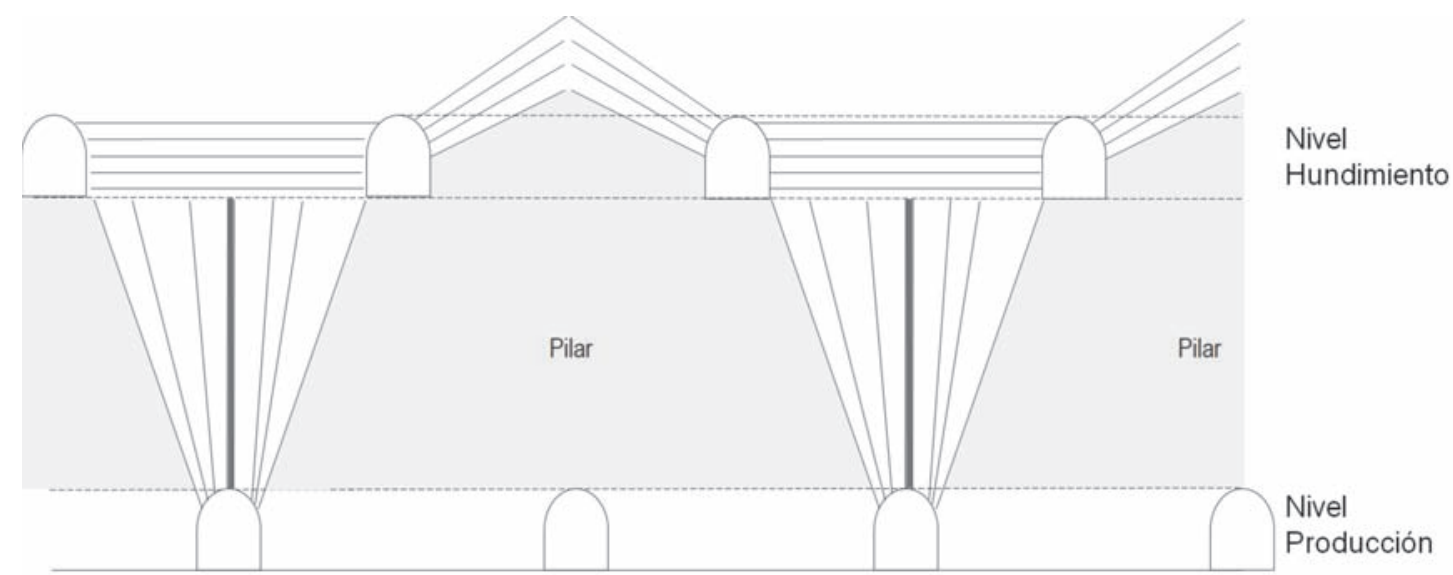

Figura 11. Socavación inclinada angosta.

Figure 11. Narrow inclined scour.

ques. Sin embargo, el concepto Panel Caving se basa en un crecimiento continuo en incrementos discretos para mantener una cantidad constante de área productiva. Esto se hace balanceando cuidadosamente el área recién incorporada y agotada.

Para asegurar una explotación sostenible en el mediano y largo plazo mediante el método de Block/Panel Caving esta requiere de reglas del caving como base de la operación. Estas reglas permiten generar una estrategia de minería definida que es corregida realizando seguimiento y observación del comportamiento en terreno.

En términos generales se puede identificar cuatro categorías de condiciones de extracción (Figura 12)

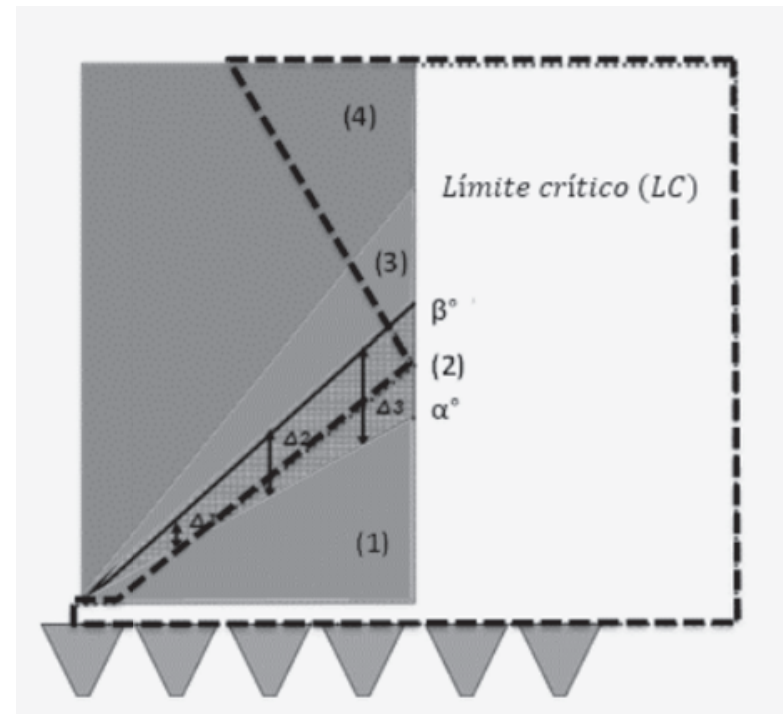

pero se resguarda el seguimiento y control para las dos condiciones de borde:

- En el caso de zonas con un ángulo de extracción bajo, se recomienda aplicar una extracción constante y un alto cumplimiento al dibujo de velocidad máxima que no incorpore una nueva área de producción, hasta que sea posible obtener una geometría de cavidad admisible.

- En el caso de zonas con extracción excedida, incorpore nueva área a la producción en zonas donde se identifica este problema, distribuyendo la extracción de manera que pueda estimular el crecimiento de nuevos puntos.

Para la secuencia de socavación se llevará una

$$
L C=P \text { lano } \beta^{\circ}+\left(\text { Plano } \beta^{\circ}-P \text { lano } \alpha^{\circ}\right)
$$

(1) Ángulo bajo de extracción

(2) Ângulo de extracción bueno

(3) Ángulo medio de extracción

(4) Ángulo de extracción excedido

Figura 12. Categorías de condiciones de extracción. Figure 12. Categories of extraction conditions. 
geometría cóncava en la dirección del frente de hundimiento, es decir, el centro de curvatura debe estar dentro de la zona hundida. El frente de hundimiento debe orientarse en $90^{\circ}$ (con una tolerancia de $+/-30^{\circ}$ ) respecto a la dirección del esfuerzo principal mayor, ya que, la zona de concentración de esfuerzos delante del frente de socavación será más benigna (menores zonas en tracción). El frente de hundimiento debe orientarse lo más perpendicular posible a las estructuras principales y contactos litológicos, con el objetivo de reducir la concentración de esfuerzos entre dichas discontinuidades y el área hundida.

Se debe tratar de minimizar la formación de cuñas inestables entre la línea de hundimiento y los planos de debilidad (fallas, contactos y área hundida). También se debe evitar frentes con geometría que formen ángulos rectos o agudos, ya que estos generan concentraciones de esfuerzos, aumentando la probabilidad de ocurrencia de daños. Dejar frentes dinámicos detenidos por prolongado tiempo (especialmente sobre infraestructura relevante, como piques, entre otros) puede producir un deterioro progresivo en el tiempo del macizo rocoso delante del frente de socavación.

El avance de la extracción debe ser concordante con la socavación en orientación y geometría (válido para todo método de explotación). Considerar un ángulo de quiebre desde el frente de extracción de $65^{\circ}$ a $75^{\circ}$, condición que se regulariza con las velocidades de extracción y la incorporación de área a la producción. El ángulo de quiebre se esquematiza en Figura 13.

En resumen, el control del crecimiento sigue similares reglas que la planificación de mediano plazo, las que son resumidas a continuación (ver Figura 14):
- Las contingencias operacionales podrían afectar la forma y el crecimiento del frente, cambiando $D(t)$.

- La disponibilidad del área no es la media, cambiando $\mathrm{H}(\mathrm{t})$.

- Las contingencias operativas y la disponibilidad de áreas reales pueden producir desviaciones de la geometría de la cueva de planificación.

- En caso de desviaciones graves, el ángulo de extracción (a) puede alcanzar un valor no admisible, lo que aumenta el riesgo en la actividad minera.

\section{Recientes modificaciones al método}

Si bien el método de Block Caving continuamente se ha ido modificando adaptándose a los nuevos desafíos que van surgiendo, existen dos metodologías que se han estudiado y aplicado en la última década que buscan enfrenar las problemáticas más recientes del método, conocidas como: Minería continua y Macro bloques.

\section{Minería continua}

El concepto de minería continua en explotaciones de Block Caving se ha venido desarrollando durante la última década en Chile por parte de Codelco con el objetivo de lograr incrementar las velocidades de extracción, si bien existen pruebas a escala de laboratorio y piloto, no se ha aplicado a gran escala. El sistema consiste en realizar de manera continua la

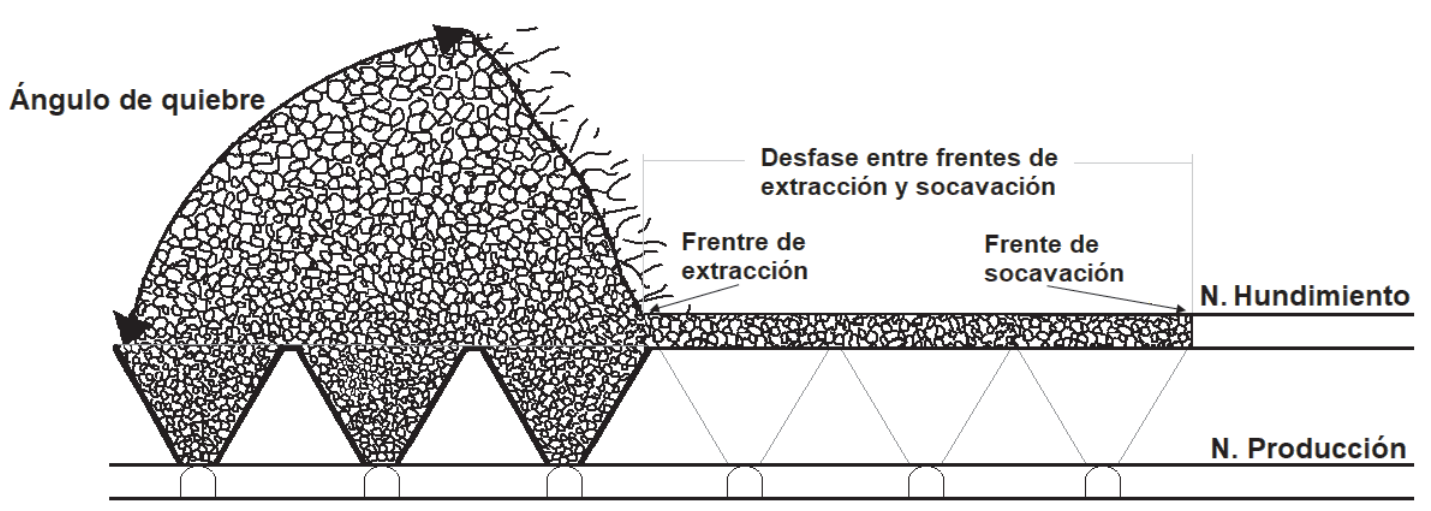

Figura 13. Ángulo de quiebre, método de Block/Panel Caving.

Figure 13. Break angle, Block / Panel Caving Method. 
Perfil de Velocidad Extracción

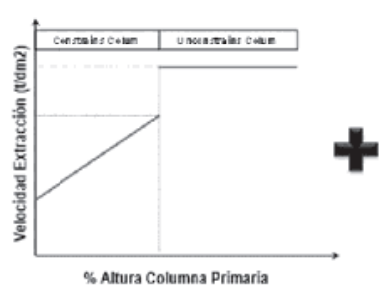

$H(t)$

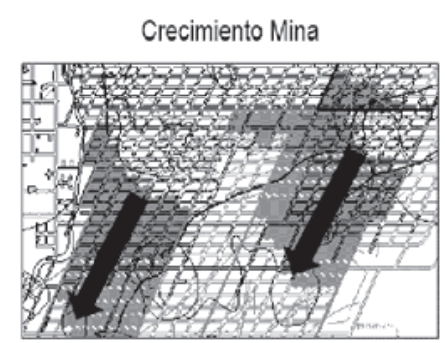

$\mathrm{D}(\mathrm{t})$

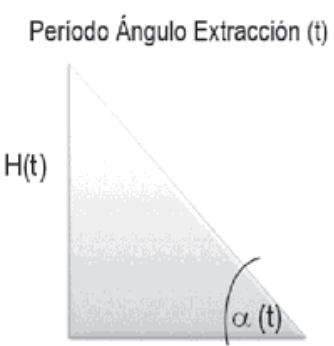

$\mathrm{D}(\mathrm{t})$

Figura 14. Reglas del caving como base para una operación sostenible.

Figure 14. Cave rules as a basis for a sustainable operation.

explotación de un yacimiento masivo de Caving, donde se pueda extraer simultáneamente de más de un punto de extracción asociado a la misma calle con el fin de alcanzar mayores productividades, automatizando el sistema de manejo de materiales a través equipos Dozer (dosificador) y Panzer (transportador blindado), indicados en Figura 15. El Dozer es un sistema carguío que funciona como pivote ubicado bajo la batea de extracción empujando el mineral hacia una calle de producción donde se encuentra el Panzer, el cual es una cinta transportadora metálica capaz de manejar fragmentos gruesos que luego son descargados sobre un equipo de machaqueo (tritura- ción). Así, el sistema requiere alto grado de automatización para generar la interacción entre los Dozers ubicados en una calle que descargan sobre el mismo Panzer. Se espera con esta dinámica alcanzar altos ritmos de producción cuando se tiene una fragmentación favorable.

El sistema requiere una mayor cantidad de desarrollos que un Block Caving convencional con LHDs, en particular por el desarrollo de galerías de servicio requeridas para la mantención de los equipos ubicados bajo los puntos de extracción, esto eleva consecuentemente los costos de desarrollos. Este sistema a la fecha se ha aplicado a escala mina en sectores

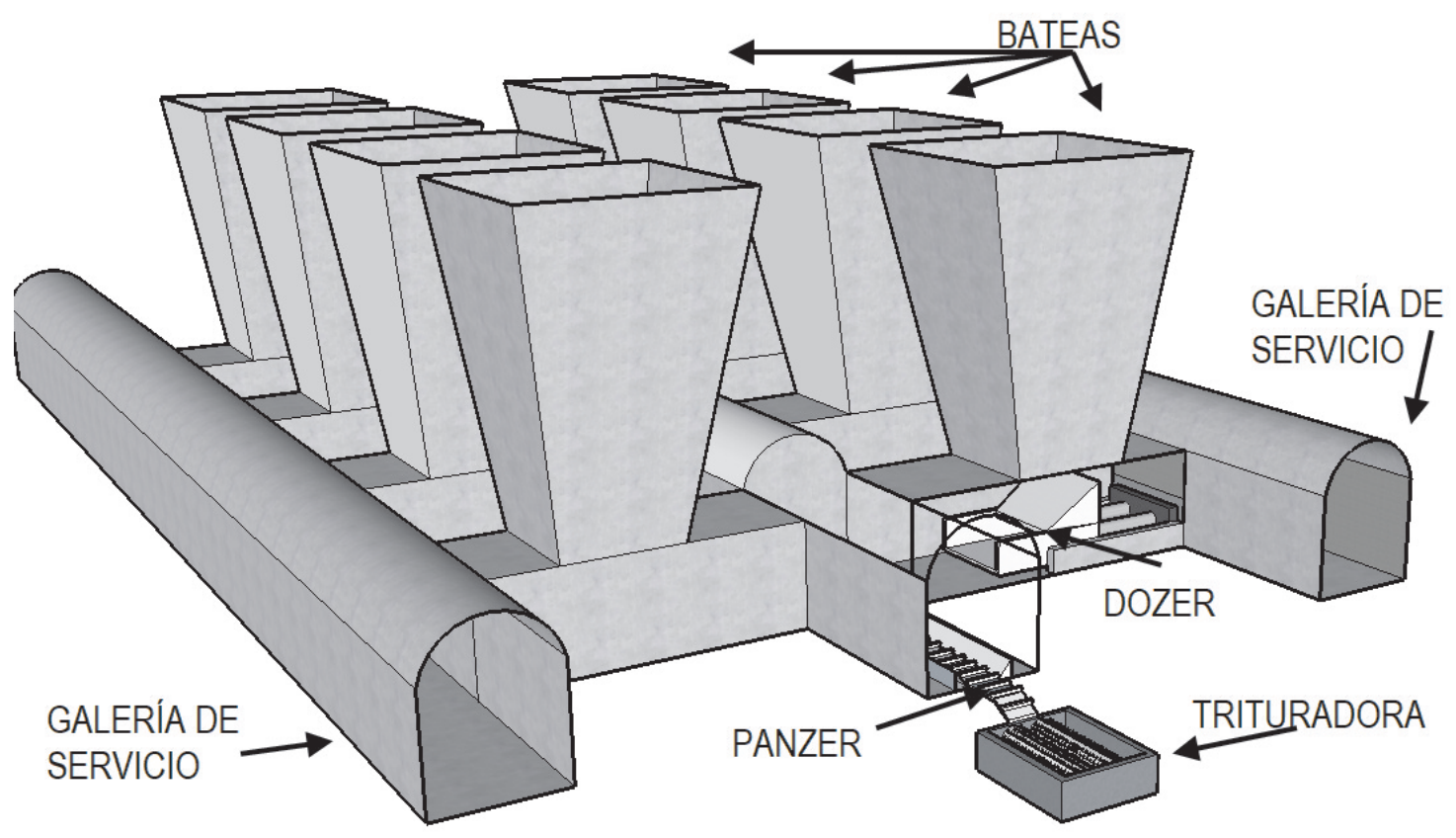

Figura 15. Esquema general sistema de Minería Continua (después de Encina et al., 2008).

Figure 15. General scheme of Continuous Mining system (Encina et al., 2008). 
específicos en algunas operaciones de hundimiento para su evaluación como es el caso de División Andina.

\section{Macro Bloques}

El concepto de Macro-bloques fue analizado y desarrollado como una extensión del método clásico de Block Caving, incorporando las últimas experiencias de Codelco en la explotación de Block/Panel Caving, especialmente en la gestión de operaciones mineras, geomecánica y temas relacionados con la geometría del cuerpo mineralizado.

Esta nueva configuración del método será aplicada en el proyecto Mina Chuquicamata Subterránea, que considera en su planificación una alta tasa de producción de 140.000 tpd. Para alcanzar este objetivo, es necesario preparar un área inicial de $102.000 \mathrm{~m}^{2}(400$ puntos de extracción) para el primer año productivo y

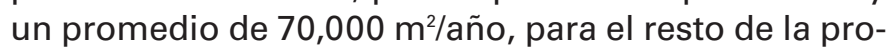
ducción horizonte (Fuentes and Villegas 2014).

Considerando estos enormes requisitos de preparación y la experiencia de las operaciones de Codelco sobre cómo lidiar con las interferencias producidas por las actividades simultáneas de preparación y explotación, se definió la implementación de un método de configuración de explotación que facilitara el manejo de interferencias durante el ciclo de minería. Este método divide o separa el área en preparación del área que se está socavando y del área bajo explotación (Araneda and Sougarret 2007). Cada
Macro-bloque es un área productiva independiente, como se presenta en la Figura 16.

La explotación se lleva a cabo de manera discreta e independiente a través de grandes áreas productivas $\left(24,000-39,000 \mathrm{~m}^{2}\right)$ dejando un pilar entre cada Macro-bloque para evitar problemas geotécnicos de sobrecarga de esfuerzos inducidos, que en el peor de los casos genera un colapso de un gran área productiva y entrada prematura de dilución, frecuentes en minería de Panel Caving.

La configuración por Macro-bloques toma la experiencia vivida en operaciones de hundimiento que han sufrido colapsos geomecánicos donde la solución al problema fue abandonar el área colapsada y reiniciar el caving (Pardo y Villascusa 2012), dejando un "pilar" de material remanente de separación entre el área afectada y el área de producción "nueva".

Para maximizar la productividad de cada Macrobloque, cada uno fue diseñado como una unidad de explotación independiente, con sus propios piques de traspaso de mineral, sistema de chancado y accesos. En otras palabras, cada Macro-bloque se considera como una mina independiente que produce mineral y los entrega al sistema principal de transporte (Fuentes and Villegas 2014)

Cada Macro-bloque debe tener un área suficiente para iniciar un nuevo el hundimiento, y además, requiere de una política de control de tiraje rigurosa, basada en una estricta planificación y extracción, para evitar o controlar el riesgo de entrada de dilución lateral de Macro-bloques vecinos.

Algunas de las oportunidades que el hundimiento por

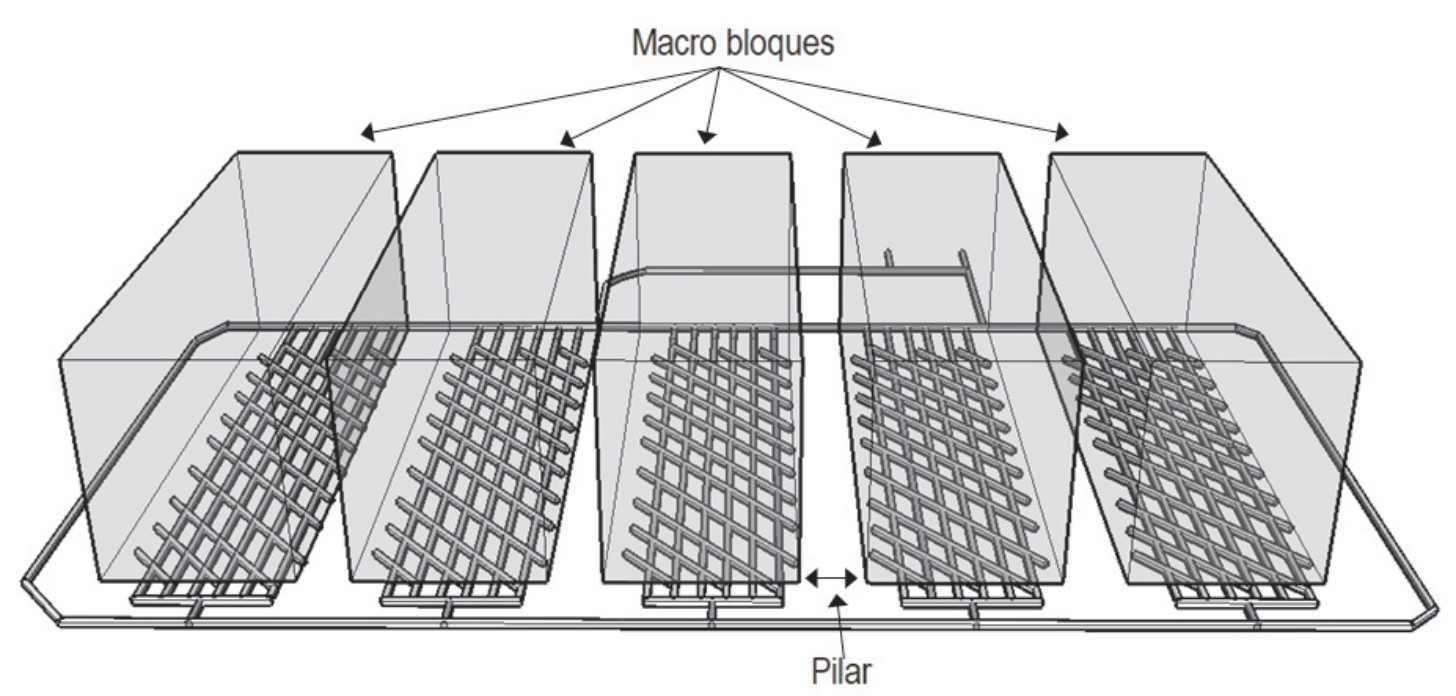

Figura 16. Explotación en configuración Macro Bloques.

Figure 16. Exploitation in Macro Blocks configuration. 
Macro-bloques ofrece a la explotación de una mina subterránea son:

- La posibilidad de concentrar actividades similares en áreas limitadas.

- Entregar a producción áreas no expuestas a interferencias de las faenas de socavación y preparación.

- Flexibilidad para adaptar la operación frente a contingencias como colapsos u otros.

- Permite incorporar cambios de diseño desde un bloque a otro (como por ejemplo la aplicación de nuevas tecnologías).

\section{Desafíos presentes y futuros: Pre acondicionamiento del macizo rocoso}

Como se mencionó en un comienzo, dentro de los principales desafíos enfrentados por las operaciones de hundimiento se encuentran los yacimientos con menores leyes, que nos obligan a operar con una mayor eficiencia y las mayores profundidades, que generan ambientes de altos esfuerzos y presencia de roca de mayor competencia, la que dificulta la dinámica del método.

Desde hace un par de décadas, se ha utilizado en minería metálica el concepto de pre acondicionamiento del macizo rocoso, el cual consiste en hacer un pre tratamiento de la roca in-situ, antes de comenzar la explotación, con el fin de debilitarla y favorecer el proceso de hundimiento, ambiente sísmico, como también lograr una mejor fragmentación durante la explotación. El método de pre acondicionamiento aplicada a macizos rocosos en minería metálica tiene su origen en la industria de hidrocarburos. Entre los métodos utilizados están el fracturamiento hidráulico (FH), la voladura confinada (VC) y la variante mixta utilizando ambos métodos. El fracturamiento hidráulico consiste en presurizar un tramo de una perforación al inyectar un fluido a presión, para iniciar una fractura de tensión en las paredes sanas de la perforación, o alternativamente extender una fractura preexistente, y así propagarla hacia el interior del macizo rocoso. En minería metálica dado que sólo se buscan generar o extender fracturas para dañar la roca, generalmente se utiliza agua sin aditivos, a diferencia de la industria de los hidrocarburos en donde se desea mantener las fracturas abiertas para mejorar la permeabilidad del yacimiento. Por otro lado, la utilización de explosivos se ha desarrollado de manera modular, agrupando de 3 a 4 pozos por módulo y mediante una voladura parcial se daña al macizo rocoso favoreciendo el quiebre natural de la roca en el proceso posterior de hundimiento. En la variante mixta, cuando se utilizan ambos métodos, se comienza con la aplicación del fracturamiento hidráulico para generar nuevos planos de debilidad que servirán para reflejar las ondas de choque de la voladura. En la actualidad el pre acondicionamiento, no sólo ha sido utilizado para favorecer la explotación del método, este ha sido aplicado también en etapas tempranas de desarrollo de labores, a través de FH y voladura en la frente (destressing), aplicados alrededor de las zonas de trabajo para alejar los altos esfuerzos de la frente. Esto último ha sido utilizado en los desarrollos más profundos de la operación El Teniente, metodología que ha sido aplicada anteriormente con los principales ejemplos realizados en minas canadienses de gran profundidad.

La aplicación de estos métodos de pre acondicionamiento del macizo rocoso en operaciones de Block Caving ha sido cuantificada por diversas operaciones en las cuales se ha podido observar ciertas ventajas de su aplicación destacando entre ellas una mejor fragmentación de la roca presente en los puntos de extracción, una sismicidad favorable y un hundimiento progresivo (Jeffrey et al. 2010, Pfitzner et al. 2010, Castro et al. 2014, Brzovic et al. 2014).

\section{Conclusiones}

Si bien los métodos de explotación masivos están asociados a menores costos operacionales, no siempre resulta evidente su explotación, menos aún, cuando las condiciones geomecánicas en las cuales se desarrolla el método van siendo cada vez más adversas lo que nos obliga a adaptarnos de manera eficiente para asegurar el éxito de la operación. Las principales modificaciones en la actualidad se han podido observar en el presente artículo, tanto en la aplicación de nuevas metodologías, como también en la implementación variantes de la explotación.

Las variantes de explotación presentadas, Minería continua y Macro bloques, fueron creadas con diferentes propósitos, la primera buscando aumentar la productividad del método de hundimiento, realizando una extracción continua desde varios puntos de extracción asociados a una calle, de esta forma, la extracción no estaría limitada a la productividad de un LHD por calle. Para considerar la implementación de esta metodología, se debe tener una calidad de macizo regular, el cual hunda rápidamente y con una buena fragmentación, para permitir mayores velocidades de hundimiento y menores interrupciones por colgaduras y/o sobre-tamaños en las bateas y puntos de extracción. La variante de Macro bloques surge 
como opción para entregar una mayor robustez al método, dada las condiciones particulares de inestabilidad en las que se enfrenta el proyecto Mina Chuquicamata Subterránea operación que plantea este nuevo diseño, donde la presencia de una falla de gran extensión al igual que encontrarse situado bajo una corta minera (rajo) de grandes dimensiones genera una condición crítica, sumado a esto se busca lograr en el proyecto grandes capacidades productivas, lo que lleva a plantear un sistema robusto, con grandes áreas en explotación y la presencia de pilares de protección.

Los métodos de hundimiento siguen siendo una opción viable y preferible cuando se buscan lograr grandes ritmos de explotación a un bajo costo en yacimientos masivos que no son rentables mediante una extracción superficial. El continuo cambio en las condiciones en las cuales se desarrollan los métodos de hundimiento, ha llevado a una adaptación sistemática para cumplir con los objetivos particulares de cada proyecto, permitiendo el desarrollo e implementación de nuevas tecnologías y dinámicas de explotación.

\section{Referencias}

Araneda, O. and Sougarret, A. 2007. 'Lessons learned in cave mining: 1997 - 2007', International Symposium on Block and Sub-Level Caving Cave Ming Keynote address. The Southern African Institute of Mining and Metallurgy, South Africa, pp. 57-71.

Bartlett, P.J. and Croll, A. 2000. Cave Mining at Premier Diamond Mine, Proceding of Massmin 2000, pp 227-235.

Beck, D., Arndt, S., Thin, I., Stone, C. and Butcher, R. 2006. A conceptual sequence for a block cave in an extreme stress and deformation environment. Procedings of Deep and High Stress Mining 2006.

Beus, M.J., Pariseau, W.G., Stewart, B.M. and Iverson, S.R. 2001. Design of Ore Passes. In: Hustrulid, W.A. and Bullock, R.L. (eds.), Underground Mining Method, 627634.

Briceño, M., Adams, M. y Castro, R. 2016. Estimación de la Humedad y Profundidad del Agua Barro en Block Caving. $1^{\text {er }}$ Congreso Internacional en Minería Subterránea: UMining 2016, Santiago, Chile, 324-338.

Brown, E.T. 2007. Block Caving Geomechanics: International Caving Study 1997-2004. Julius Kruttschnitt Mineral Research Centre, The University of Queensland, Australia.

Brunton, I., Lett, J. and Thornhill, T. 2016. Fragmentation prediction and assessment at the ridgeway deeps and Cadia east cave operations. $7^{\text {th }}$ International Conference \& Exhibition in Mass Mining: Massmin 2016. The Australasian Institute of Mining and Metallurgy, Sydney, Australia, 151-160.

Butcher, R., Joughin, W. and Stacey, T.R. 2000. A Booklet on Methods of Combating Mudrushes in Diamond and
Base Metal Mines. Safety in Mines Research Advisory Committee.

Brzovic, A., Hurtado, J.P. and Marin, N. 2014. Intensity rock mass preconditioning and fragmentation performance at the El Teniente mine, Chile. $3^{\text {rd }}$ international symposium on block and sublevel caving: Caving 2014, Santiago, Chile, 547-557.

Calder K., Townsend P. y Russell F., 2000. The Palabora Underground Mine Project, Procedings of Massmin 2000, pp 219-225.

Carter, T. G. (1992). A new approach to surface crown pillar design. In Proc. 16th Can. Rock Mechanics Symposium, Sudbury (pp. 75-83).

Castro, R. 2006. Study of the mechanisms of gravity flow for block caving. PhDThesis, The University of Queensland, Australia.

Castro, C., Báez, F., Arancibia, E. and Barrera, V. 2014. Study of the impact of rock mass preconditioning on a Block Caving Mine Operation. $3^{\text {rd }}$ international symposium on block and sublevel caving: Caving 2014, Santiago, Chile, 515-524.

Castro, R.L. and Paredes, P.S. 2014. Empirical observations of dilution in panel caving. Journal of the Southern African Institute of Mining and Metallurgy, 114 (6), 455-462.

Castro, R. and Pineda, M. 2015. The role of gravity flow in the design and planning of large sublevel stopes. Journal of the Southern African Institute of Mining and Metallurgy, 115 (2), 113-118.

Castro, R., Gómez, R. and Hekmat, A. 2016. Experimental quantification of hang-up for block caving applications. International Journal of Rock Mechanics and Mining Sciences, (85), 1-9.

Cochilco, 2014. Factores Clave para el Desarrollo de la Minería en Chile. Ministerio de Minería, Chile.

Codelco, División El Teniente. 2010. Definición de matriz de criticidad para control de agua-barro. Superintendencia Gestión Producción,

Codelco, 11/01/18, https://www.codelco.com

De Nicola, R. and Fishwick, M. 2000. 'An Underground Air Blast' - Codelco Chile - Division Salvador. Massmin 2000, Brisbane, Australia, 279-288.

Duplancic, P. and Brady, B.H. 1999. Characterisation of caving mechanisms by analysis of seismicity and rock stress. 9th ISRM Congress. International Society for Rock Mechanics, 1049-1053.

Eadie, B. 2003. A framework for modelling fragmentation in block caving. PhDThesis, The University of Queensland, Australia.

Emol, 2017. Mapa: Cuánto cuesta la energía eléctrica de uso residencial en Chile y el mundo. 19/07/17, http://www.emol.com/noticias/Economia

Encina, V., Baez, F., Geister, F. and Steinberg, J. 2008. Mechanized continuous drawing system: A technical answer to increase production capacity for large block caving mines. 5th International Conference \& Exhibition on Mass Mining: Massmin2008, Luleå, Sweden, 553-562.

Flores, G. 2005. Rock mass response to the transition from open pit to underground cave mining. PhD Thesis, JKMRC School of Engineering the University of Queensland. 
Flores, G. 2014. Future challenges and why cave mining must change. $3^{\text {rd }}$ international symposium on block and sublevel caving: Caving 2014, Santiago, Chile, 23-52.

Flores, G. and Karzulovic, A. 2002. Benchmarking Report. Geotechnical guidelines for a transition from open pit to underground mining. International caving study II, $J K R M C$, University of Queensland.

Fuentes, S. and Villegas, F. 2014. Block caving using macro blocks. $3^{\text {rd }}$ International symposium on block and sublevel caving: Caving 2014, Santiago, Chile, 23-52.

Gómez, R., Castro, R.L., Casali, A., Palma, S. and Hekmat, A. 2017. A Comminution Model for Secondary Fragmentation Assessment for Block Caving. Rock Mechanics and Rock Engineering, 50 (11), 3073-3084.

Hadjigeorgiou, J. and Lessard, J.F. 2007. Numerical investigation of ore pass hang-up phenomena. International journal of rock mechanics \& mining sciences, 44 (6), 820834.

Hamrin, H. 2001. Underground mining methods and applications. In: Hustrulid, W.A. and Bullock, R.L. (eds.), Underground Mining Method, 3-14.

Hashim, M. 2011. Particle Percolation in block Caving mines. PhD Thesis, Australia, University of New South Wales, Australia.

Jakubec, J. 2014. Fragmentation estimates using BCF software - Experiences and pitfalls. 3rd international symposium on block and sublevel caving: Caving 2014, Santiago, Chile, $191-200$.

Jeffrey, R.G., van As, A., Zhang, X., Bunger, A.P. and Chen, Z.R. 2010. Measurement of hydraulic fracture growth in a naturally fractured orebody for application to preconditioning. Second International Symposium on Block and Sublevel Caving: Caving 2010, Perth, Australia, 647-662.

Karzulovic, A. 1998. Evaluación Geotécnica Métodos de Socavación Previa y Avanzada Mina El teniente, estudio DT - CG - 98 - 003, División EITeniente, Codelco-Chile.

Kvapil, D.R. 2008. Gravity flow in sublevel and panel caving - A common sense approach. Luleå University of Technology.

Labbé, E. 2014. Catastro de oportunidades I\&D en innovación tecnológica para minería de block/panel caving. Memoria de título, Universidad de Chile, Chile.

Lang, B. 1994. Span design for entry-type excavations. MSc Thesis, University of British Columbia, Canada.

Laubscher, D. 1994. Cave mining - The state of the art. International Journal of Geomechancis and Mining Sciences. 94(10), 279-293.

Laubscher, D.R. 2000. Block caving manual. Prepared for the International Caving Study, JKMRC and Itasca Consulting Group, Brisbane.

Lorig, L. 2004. Understanding gravity flow for mix and dilution modeling at Henderson Mine. MassMin 2004, Santiago, Chile, 231-237.

Mathews, K. E., Hoek, E., Wyllie, D. C., and Stewart, S. B. V. (1981). Prediction of stable excavation spans at depths below $1000 \mathrm{~m}$ in hard rock mines. CANMET Report, DSS Serial No. OSQ80-00081.

Ministerio de Minería, 2016. Cuenta Pública 2016.

Pardo, C. and Villascusa, 2012. Methodology for back analysis of intensive rock mass damage at the Teniente Mine, 6th International Conference \& Exhibition on Mass Mining, Sudbury, Canada.

Pfitzner, M.J., Westman, E., Morgan, M. and Finn, D. 2010. Estimation of rock mass changes induced by hydraulic fracturing and cave mining by double difference passive tomography. Second International Symposium on Block and Sublevel Caving: Caving 2010, Perth, Australia, 677684.

Pierce, M. 2009. A model for gravity flow of fragment rock in block caving mines. PhD Thesis, The University of Queensland, Australia.

Pierce, M.E., Waetherley, D.K. and Kojovic, T.A. 2010. Hybrid methodology for secondary fragmentation prediction in cave mines. Second International Symposium on Block and Sublevel Caving: Caving 2010, Perth, Australia, 567582.

Rojas E., Molina R., Bonani A. y Constanzo H. 2000. The preundercut caving method at El Teniente Mine, Codelco Chile, Procedings of Massmin 2000.

Sahupala, H., Brannon, C., Annavarapu, S. and Osborne, K. 2008. Recovery of extraction level pillars in the Deep Ore Zone (DOZ) block cave, PT Freeport Indonesia. 5th International Conference \& Exhibition on Mass Mining: Massmin2008, Luleå, Sweden, 191-202.

Samosir, E., Basuni, J., Widijanto, E. and Syaifullah, T. 2008. The Management of Wet Muck at PT Freeport Indonesia's Deep Ore Zone Mine. 5th International Conference \& Exhibition on Mass Mining: Massmin2008, Lulea, Sweden, 323-332.

Silveira, A.C. 2004. Undercutting at E26 lift 2 Northparkes. Procedings of Massmin 2004, pp 410 - 414.

Susaeta, A., Rubio, E., Pais, G. and Enriquez, J. 2008. Dilution behaviour at Codelco panel cave mines. 5th International Conference \& Exhibition on Mass Mining: Massmin2008, Lulea, Sweden, 167-178.

Syaifullah, T., Widijanto, E. and Srikant, A. 2006. Water Issues in DOZ Block Cave Mine, PT Freeport Indonesia. Water in Mining Conference.

Trueman, R., Pierce M. y Wattimena, R. 2002. Quantifying stresses and support requirements in the undercut and production level drifts of block and panel caving mines.

“Undercutting Workshop", Phoenix, 2008. Rio Tinto, CODELCO, Freeport McMoRan. Presentación.

Vallejos, J., Basaure, K., Palma, S. and Castro, R.L. 2017. Methodology for evaluation of mud rush risk in block caving mining. Journal of the Southern African Institute of Mining and Metallurgy, 117 (5), 491-497.

Wattimena, R.K. 2003. Designing undercut and production level drifts of block caving mines. PhD Thesis, JKMRC School of Engineering. The University of Queensland.

Recibido: diciembre 2017

Revisado: febrero 2018

Aceptado: junio 2018

Publicado: marzo 2019 